\title{
Direct computation of instability points for contact problems
}

\author{
H. Tschöpe, E. Oñate, P. Wriggers
}

Abstract The extended system is known as a reliable algorithm for the direct computation of instability points on the equilibrium path of mechanical structures. This article describes the application of the extended system as critical point computation method to mechanical contact problems. In this type of problems inequality constraints have to be considered. Moreover a prediction method based on the extended system algorithm is presented which allows the detection of favorable starting values for a critical point computation on the equilibrium path.

Keywords Critical points, Contact, Extended system

\section{1}

\section{Introduction}

In engineering exact knowledge of stability behavior of mechanical structures is important since it reveals information about structural failure. Commonly for the tracing of non-linear equilibrium paths so called arclength procedures are applied (Wemper, 1971; Ricks, 1972). In combination with finite elements this is a powerful tool for the calculation of load-deflection paths. For quite some time the detection of critical points was left to test functions, such as e.g., the determinant or the smallest eigenvalue of the tangent stiffness matrix, that were computed accompanyingly to the path tracing. A thorough distinction of several types of these critical points is given in Jepson and Spence (1985).

With the appearance of direct methods in mathematics, see Abbott (1978); Seydel (1979) the computation of critical points simplified considerably. In engineering these methods appeared with the articles of Wriggers et al. (1988) and Wriggers and Wagner (1989). Some enhancements of the method were proposed later in Wriggers and Simo (1990). Starting with simple truss elements in the

H. Tschöpe $(\bowtie)$, P. Wriggers

Institut für Baumechanik und Numerische

Mechanik University of Hannover,

Appelstr. 9A, 30167 Hannover, Germany

E. Oñate

E.T.S. Ingenieros de Caminos, Canales y Puertos, Universidad Politécnica de Cataluña Edificio C 1, Gran Capitan s/n, 08034 Barcelona, Spain

Dedicated to the memory of Prof. Mike Crisfield, for his cheerfulness and cooperation as a colleague and friend over many years. beginning the method was applied to more complex two dimensional structures involving solid elements. In recent years the prediction method of Oñate and Matias (1996) and Oñate et al. (2000) was added to the set of critical point detection methods.

In this article the application of the extended system method to a new class of problems namely mechanical contact problems is presented. The main difficulty that arises in this context are the inequality constraints that have to be considered. These constraints originate from the non-penetration condition in contact mechanics. A rather straightforward way of the extended system application to this problem type is shown. Some numerical examples at the end of this article confirm the effectivity of this method.

A good overview on the whole subject of instability points can be found in Wagner (1991). The topic of this article is treated in more detail in Tschöpe (2001), which also contains further examples.

\section{2}

Basic equations

In this chapter the finite element formulation that is used in this work is described briefly. A general continuum mechanical formulation for isothermal, quasi-static contact problems will be derived.

\section{1}

\section{Continuum mechanics}

With the deformation energy $W$ for hyperelastic material the principle of the minimum of potential energy with respect to the reference configuration can be stated as:

$\Pi_{S}=\int_{\mathcal{B}} W \mathrm{~d} V-\int_{\mathcal{B}} \rho_{0} \hat{\mathbf{b}} \cdot \mathbf{u d} V-\int_{\partial \mathcal{B}_{\sigma}} \hat{\mathbf{t}} \cdot \mathbf{u} \mathrm{d} A \Rightarrow \operatorname{Min}$.

Besides the term for the elastic energy, terms considering the energy of applied body forces $\mathbf{b}$ and surface loads $\mathbf{t}$ have been added. The unknown displacement function is denoted by $\mathbf{u}$.

Assuming the St. Venant constitutive law, the elastic energy is $W=\frac{1}{2}(\mathbf{S E})=\frac{1}{2}(\mathbf{E}:(\mathbf{D E}))$. Here $\mathbf{S}$ is the 2. PiolaKirchhoff stress tensor, $\mathrm{D}$ is the fourth order constitutive tensor and $\mathbf{E}$ the Green-Lagrange strain tensor according to $\mathbf{E}=\frac{1}{2}\left(\mathbf{F}^{\mathrm{T}} \mathbf{F}-\mathbf{I}\right)$ with the deformation gradient $\mathbf{F}=(\mathbf{I}+\operatorname{Grad} \mathbf{u})$. Variation of (1) then yields 


$$
\begin{aligned}
\delta \Pi_{S}= & \int_{\mathcal{B}} \mathbf{E}: \mathbf{D} \delta \mathbf{E} \mathrm{d} V-\int_{\mathcal{B}} \rho_{0} \hat{\mathbf{b}} \cdot \delta \mathbf{u} \mathrm{d} V \\
& -\int_{\partial \mathcal{B}_{\sigma}} \hat{\mathbf{t}} \cdot \delta \mathbf{u} \mathrm{d} A=0,
\end{aligned}
$$

where the variation of the Green-Lagrange strain tensor is $\delta \mathbf{E}=\frac{1}{2}\left(\mathbf{F}^{\mathrm{T}} \operatorname{Grad} \delta \mathbf{u}+\operatorname{Grad}^{\mathrm{T}} \delta \mathbf{u} \mathbf{F}\right)$

and $\delta \mathbf{u}$ is the test function for which $\left\{\delta \mathbf{u} \mid \delta \mathbf{u}=\mathbf{0}\right.$ on $\left.\partial \mathcal{B}_{u}\right\}$ is required.

\section{2}

\section{Finite element method}

For the application of the finite element method the area of integration $B$ is approximated by the discretization

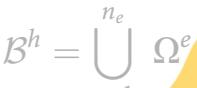

with $n_{e}$ finite elements. The displacements $\mathbf{u}$ and the virtual displacements $\delta \mathbf{u}$ are then interpolated on the elemental level by a sum of shape functions $N$ and nodal values $\mathbf{v}$ :

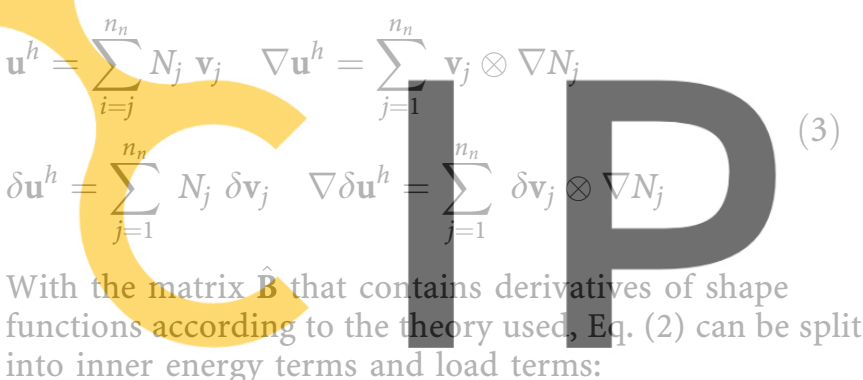
into inner energy terms and load terms:

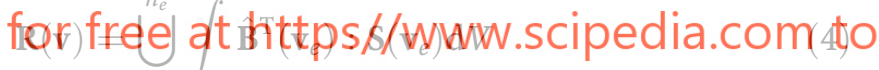 \\ $\mathbf{P}(\mathbf{v})=\bigcup_{e=1}^{n_{e}}\left[\int_{\Omega^{e}} \rho \hat{\mathbf{b}} \mathrm{d} V+\int_{\partial \Omega^{e} \sigma} \hat{\mathbf{t}} \mathrm{d} A\right]$.

This leads finally to the set of equations that has to be solved for the unknown nodal displacements

$\mathbf{G}_{S}(\mathbf{v})=\mathbf{R}(\mathbf{v})-\lambda \mathbf{P}=\mathbf{0}$,

where the load scaling parameter $\lambda$ for equilibrium path computations has already been introduced. Commonly this equation set is solved with the Newton-Raphson method. Due to the complexity of the governing equations the load-deflection path exhibits slopes so that arclength methods for the curve tracing become necessary, see Ricks (1972), Crisfield (1981), Ramm (1981) and Schweizerhof and Wriggers (1986). In this work the arclength method with an updated normal plane control equation is applied.

\section{3}

\section{Contact}

For contact modeling a two dimensional frictionless normal contact formulation is used. Therefore the masterslave concept of Hallquist (1979) is adopted, where one of the bodies is chosen as the master $\mathcal{B}^{M}$, the other as the slave $\mathcal{B}^{S}$. In numerical contact mechanics this is a rather common model, that can be found in several articles, e.g. Curnier (1984), Wriggers and Miehe (1992) and Laursen and Simo (1993).

For a given slave node $\mathbf{x}^{S}$ on $\partial \mathcal{B}_{c}^{S}$ a distance function $d\left(\xi_{\alpha}\right)$ to the parameterized master surface $\mathbf{x}^{M}\left(\xi_{\alpha}\right)$ can be defined. The corresponding master point $\mathbf{x}^{M}\left(\bar{\xi}_{\alpha}, t\right)$ for the slave point $\mathbf{x}^{S}$ can be found minimizing this distance function

$d\left(\xi_{\alpha}\right)=\left\|\mathbf{x}^{S}-\mathbf{x}^{M}\left(\xi_{\alpha}\right)\right\| \Rightarrow$ MIN with $\alpha=1,2$.

This leads to the local gap function $g_{N}$, which permits to distinguish between the different contact states:

$g_{N}=\left[\mathbf{x}^{S}-\overline{\mathbf{x}}^{M}\right] \cdot \overline{\mathbf{n}}$

The outward directed normal vector $\overline{\mathbf{n}}$ is obtained from the convective coordinate system of the surface discretization

$$
\overline{\mathbf{a}}_{\alpha}=\mathbf{x}_{, \alpha}\left(\bar{\xi}_{\alpha}\right), \quad \overline{\mathbf{n}}=\frac{\overline{\mathbf{a}}_{1} \times \overline{\mathbf{a}}_{2}}{\left\|\overline{\mathbf{a}}_{1} \times \overline{\mathbf{a}}_{2}\right\|}
$$

Since negative values of $g_{N}$ would mean a penetration of the two bodies, which is not allowed, the constraint $g_{N} \geq 0$ yields. For the incorporation of this constraint in the set of Eq. (6) a penetration function

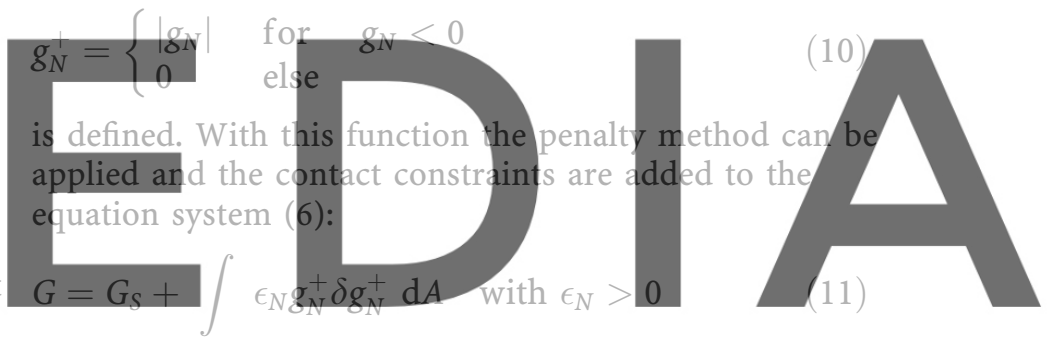

\section{downloadnthenversion without the watermark} The penalty method does not solve the problem exactly, it only regularizes it. The great advantage of this method is the ease of implementation in existing finite element program codes. However due to the regularization tiny rest penetrations remain after the problem is solved. The penalty parameter $\epsilon_{N}$ has to be chosen properly, so that on one side ill conditioning of the equation system and on the other side too large rest penetration do not occur.

For the numerical examples computed in this work a node to segment contact discretization has been used. In general the description of contact presented can be found in Wriggers (1995) and several other publications.

\section{3}

\section{Computation of critical points}

For the calculation of critical points on the equilibrium path of a mechanical structure two types of methods, namely direct and indirect methods, exist. Here a direct method, the extended system with the eigenvalue problem as additional constraint is applied to the contact problems. This chapter contains a brief description of this algorithm and in addition a prediction method derived from it. The latter can be useful in the choice of favorable starting values. 


\section{1}

\section{Extended system}

The solution of equation systems of the type (6) and (11) is accomplished with the Newton-Raphson method. This standard finite element algorithm for Eq. (11) reads as

$$
\begin{aligned}
& {\left[\mathbf{K}_{T}\left(\mathbf{v}^{i}\right)+\mathbf{K}_{c}\left(\mathbf{v}^{i}\right)\right]_{\Delta} \mathbf{v}^{i+1}=-\mathbf{G}_{S}\left(\mathbf{v}^{i}\right)-\mathbf{G}_{c}\left(\mathbf{v}^{i}\right) \text { with }} \\
& \mathbf{v}^{i+1}=\mathbf{v}^{i}+{ }_{\Delta} \mathbf{v}^{i+1} .
\end{aligned}
$$

The matrices $\mathbf{K}_{T}$ and $\mathbf{K}_{c}$ are the contributions to the global tangent stiffness matrix originating from the solid and contact terms $\mathbf{G}_{s}$ and $\mathbf{G}_{c}$ respectively:

$\mathbf{K}_{T}=\frac{\partial \mathbf{G}_{S}}{\partial \mathbf{v}}, \quad \mathbf{K}_{c}=\frac{\partial \mathbf{G}_{c}}{\partial \mathbf{v}}$

For the direct computation of instability points with the extended system, the equation system (11) is extended by a condition for the critical point. In this work the eigenvector equation in the critical point $\mathbf{K}_{T} \boldsymbol{\phi}=0$ was chosen. This procedure has been described before in literature in several publications, see e.g. Wriggers et al. (1988),

Wriggers and Wagner (1989), Wriggers and Simo (1990). With the additional constraint and a scaling equation for the eigenvector, the extended equation system is

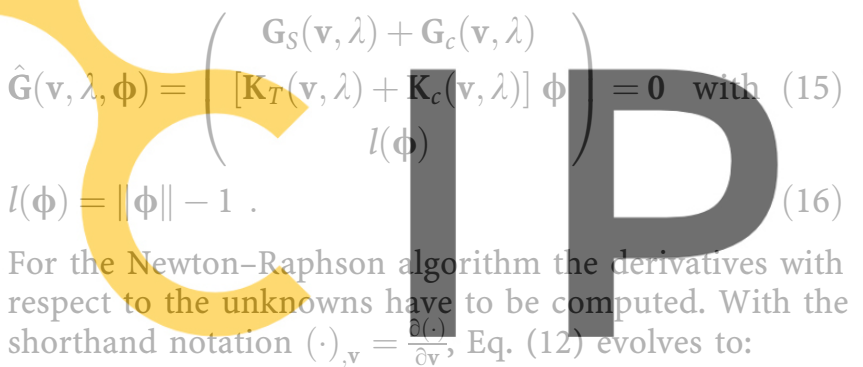
shorthand notation $(\cdot)_{\mathrm{v}}=\frac{\mathrm{\partial}(\cdot)}{\partial \mathrm{v}}$, Eq. (12) evolves to:

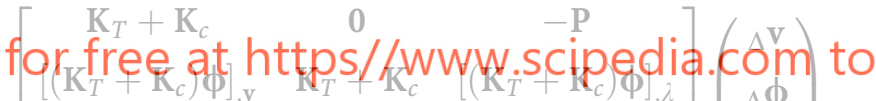

$$
\begin{aligned}
& 0^{T} \quad \frac{\phi^{T}}{\|\phi\|} \\
& =-\left(\begin{array}{c}
\mathbf{G}_{S}(\mathbf{v})+\mathbf{G}_{c}(\mathbf{v}) \\
{\left[\mathbf{K}_{T}(\mathbf{v})+\mathbf{K}_{c}(\mathbf{v})\right] \boldsymbol{\phi}} \\
\|\boldsymbol{\phi}\|-1
\end{array}\right)
\end{aligned}
$$

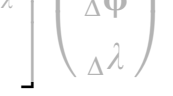

The number of unknowns has increased now to $2 n+1$ with $n$ being the degrees of freedom of the equation system (11). For the efficient solution of (17) Wriggers et al. (1988) and Wriggers and Simo (1990) proposed an algorithm with a numerical derivative as an approximation for the derivatives of $\mathbf{K}_{T}$. This algorithm is adapted to the contact problems of this article and outlined in appendix A.

In this algorithm special attention is necessary for the reassemblation of the stiffness matrix $\mathbf{K}_{c}\left(\mathbf{v}^{i}+\epsilon \boldsymbol{\phi}^{i}\right)$ in combination with the contact model used in this work. Since for each assemblation of the stiffness matrix a new mapping of slave nodes to master segments is performed in the contact algorithm, this means that $\mathbf{K}_{c}\left(\mathbf{v}^{i}\right)$ and $\mathbf{K}_{c}\left(\mathbf{v}^{i}+\epsilon \boldsymbol{\phi}^{i}\right)$ can differ substantially. To prevent this the assemblation of $\mathbf{K}_{c}\left(\mathbf{v}^{i}+\epsilon \boldsymbol{\phi}^{i}\right)$ is made basing on the master segment to slave node mapping of $\mathbf{K}_{c}\left(\mathbf{v}^{i}\right)$. Furthermore the active set of $\mathbf{K}_{c}\left(\mathbf{v}^{i}\right)$ is held fix, too. The negative effect of adhesive forces that are applied for a degree of freedom in the active set whose gap was closed in $\mathbf{K}_{c}\left(\mathbf{v}^{i}\right)$ but becomes open now in $\mathbf{K}_{c}\left(\mathbf{v}^{i}+\epsilon \boldsymbol{\phi}^{i}\right)$ are neglectable due to the low magnitude of $\epsilon \boldsymbol{\phi}$.

A proper choice of the starting values for the extended system is of great importance for the convergence of the algorithm. For the eigenvector $\phi$ several possible starting vectors exist:

$\phi_{0}= \begin{cases}1 & \text { unit vector } \\ \frac{\mathbf{v}_{t}}{\left\|\mathbf{v}_{t}\right\|} & \text { current displacement vector } \\ \frac{\mathbf{K}_{T}^{-1} 1}{\left\|\mathbf{K}_{T}^{-1} 1\right\|} & \text { 1. or higher step of an inverse iteration } \\ \phi_{0} & \text { from previous eigenvalue computations }\end{cases}$

A major advantage of this extended system algorithm is that the eigenvector $\phi$ in the critical point is computed simultaneously. This additional information is useful in bifurcation points since it shows the deformation pattern of the secondary path. This deformation pattern is needed for path switching algorithms.

\section{2}

\section{One step prediction}

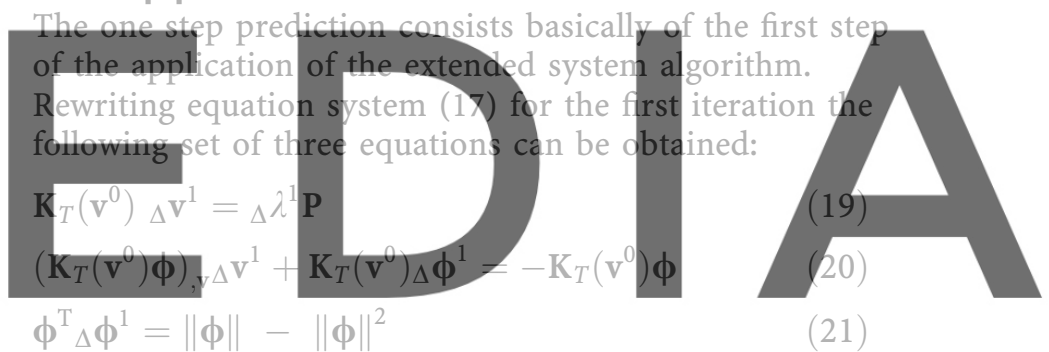

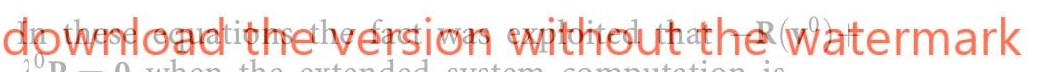
$\lambda \mathrm{P}=0$ when the extended system computation is started from a converged equilibrium point of the loaddeflection path. Solving these equations for the load update $\Delta \lambda$ yields

${ }_{\Delta} \lambda^{1}=-\frac{1}{\frac{\phi^{\mathrm{T}}}{\|\boldsymbol{\phi}\|} \mathbf{K}_{T}^{-1}\left[\left(\mathbf{K}_{T} \boldsymbol{\phi}\right)_{, \mathbf{v}} \Delta \mathbf{v}_{P}^{1}\right]} \quad$ so that

$\lambda^{1}=\lambda^{0}+{ }_{\Delta} \lambda^{1}$.

This means that an estimation of the critical load $\lambda^{1}$ can be obtained this way. The computation of the denominator in (22) can be simplified again by the numerical derivative as in the extended system algorithm (appendix A). The eigenvector $\phi$ in (22) is one of the possible starting vectors from (18).

Computing this load prediction during a path computation gives a curve of critical load estimations that indicates favorable starting values for the extended system algorithm.

\section{4}

To conclude this article two numerical examples will be shown to illustrate the application of the extended system algorithm and the one step prediction to contact problems. 
The examples have been computed with the finite element code FEAP developed by R.L. Taylor, see Zienkiewicz and Taylor (1989) for a description.

\section{1}

\section{Two arches}

The first example is the structure of an arch that has a second arch upside down on top of it. An outline containing geometrical and material data is shown in Fig. 1. A sidewards movement ( $x$-direction) of the upper arch is prevented by the boundary conditions in the corner nodes, where the unit loads are applied.

The arches were discretized with four node elements with linear shape functions. The convergence study of Fig. 2 demonstrates, that an elementation with 14000 elements is sufficient for the exactness of the numerical solution.

The results of the extended system computations are shown in Fig. 3. Two bifurcation points with $B_{1}:\left(u_{y} ; \lambda\right)=$ $-0.85 ; 17.21)$ and $B_{2}:\left(u_{\gamma} ; \lambda\right)=(-4.77 ; 75.94)$ and two limit load points with $L_{1}:\left(u_{v} ; \lambda\right)=(-12.94 ; 108.83)$ and $L_{2}:\left(u_{y} ; \lambda\right)=(-47.73 ; 42.14)$ were found successfully. The dashed lines in diagram 3 symbolize the application of the extended system. Starting point and converged end point are marked with dots.

The deformed arches and the secondary path deformation respectively are depict to each critical point. The are plots of the eigenvectors the extended system comput In Fig. 4 the curves of the calculated with the one step p plotted. Comparing these with Fig. 3 it can be seen how the load predictions indicate for each starting point to

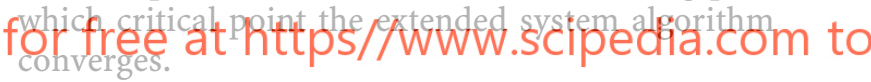

\section{2}

\section{Block pressing on arch}

The second example is the block pressing on a clamped arch, see Fig. 5 for a more thorough description. The discretization of the arch is 20 layers with 400 elements each and the block has 11 layers with 300 elements each. Except for the outer nodes with half the load, unit loads are applied. As in the previous examples a sidewards movement is prevented by fixating the $x$-displacements of the upper corner nodes of the block.

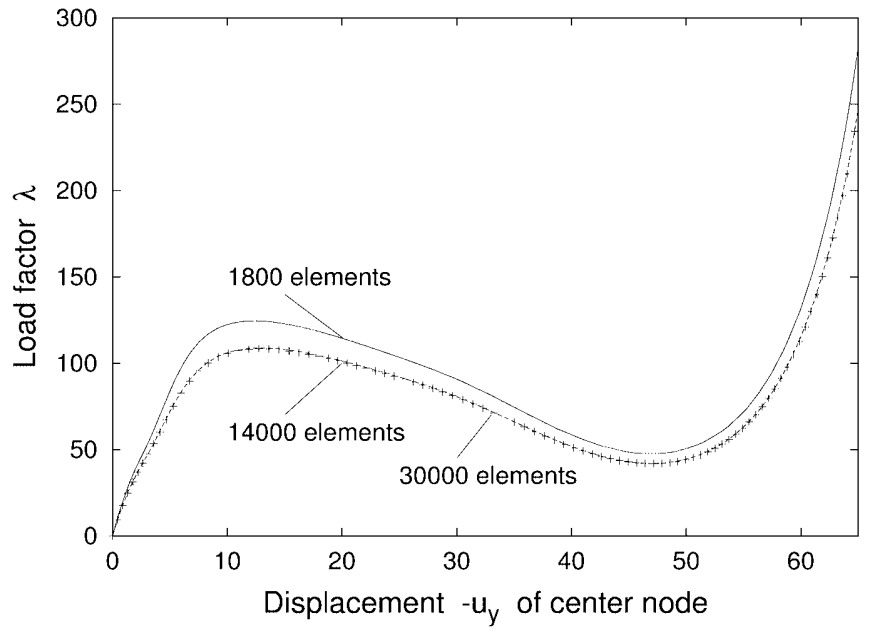

Fig. 2. Equilibrium path of the two arches structure for different discretizations

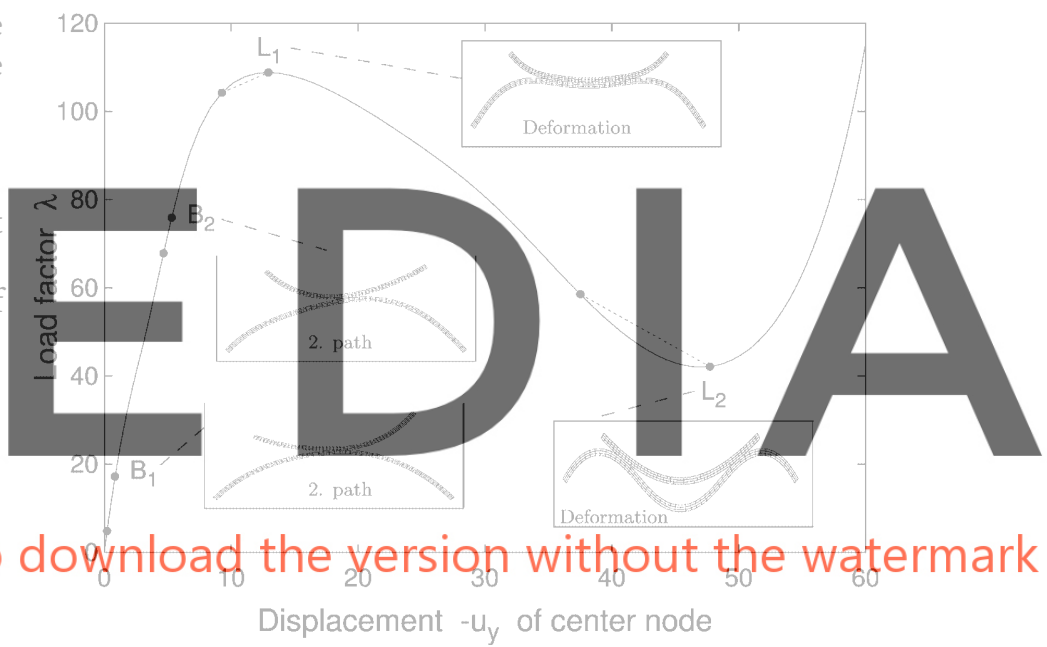

Fig. 3. Equilibrium path with critical points

As Fig. 6 demonstrates, the bifurcation point $B_{1}:\left(u_{y} ; \lambda\right)=(-23.02 ; 11.03)$ and the limit point $L_{1}:\left(u_{y} ; \lambda\right)=(-40.75 ; 126.45)$ are found by the extended system. The one step prediction in the next Fig. 7 confirms the convergence radius of the equilibrium path diagram.

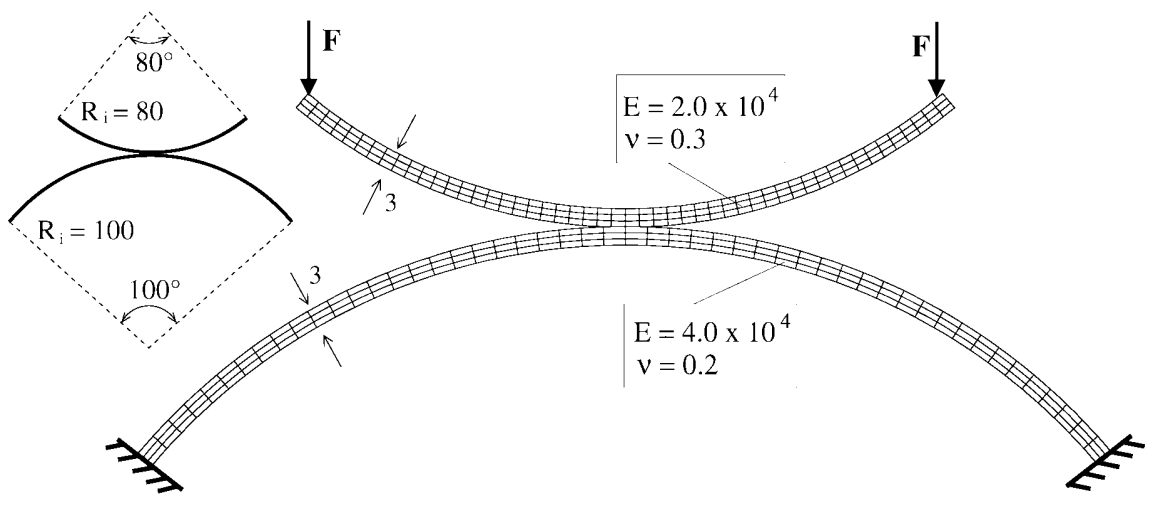

Fig. 1. Outline of the two arches 


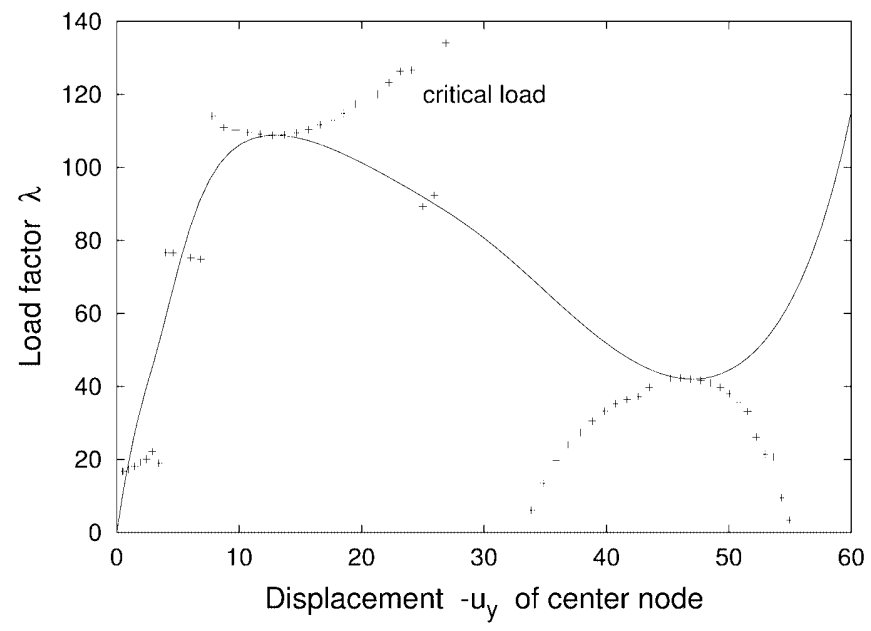

Fig. 4. One step prediction for the two arches structure

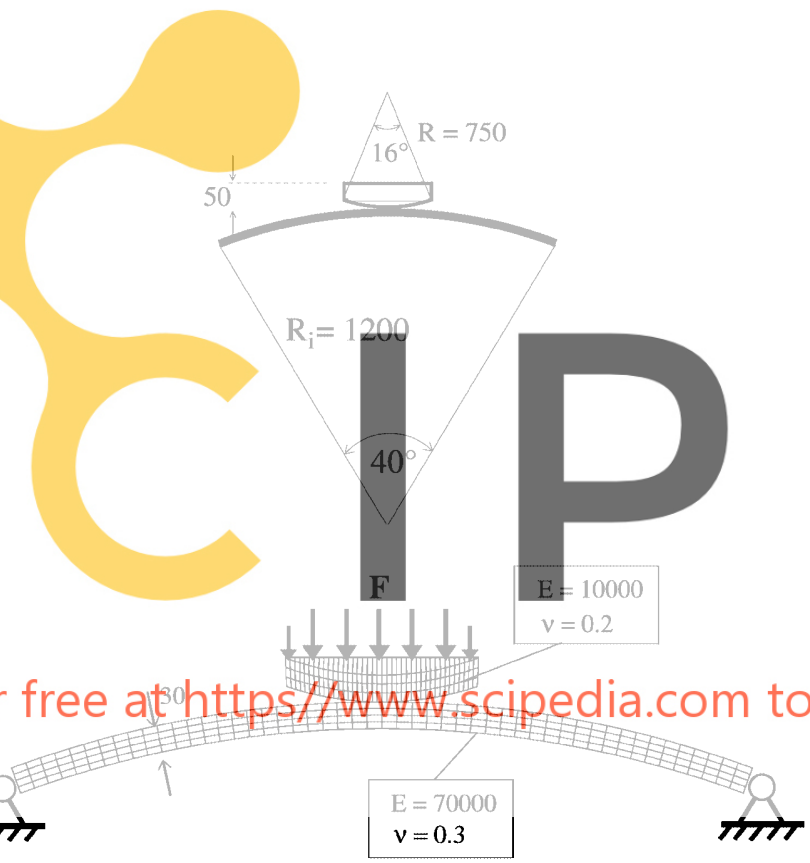

Fig. 5. Block pressing on hinged arch

\section{5}

\section{Conclusions}

This article is concerned with instability points in structural mechanics. The objective was to directly compute critical points in combination with contact problems. The difficulty that arises with these problems are the inequality constraints which have to be considered in addition.

With the extended system a reliable, well known procedure for the direct computation of critical points was extended in a rather straightforward way to the contact problems. The results with the numerical examples were very good. All critical points were computed correctly, moreover the extended system yields the eigenvector associated with an instability point.

Basing on the extended system algorithm a one step prediction method was developed, which gives a one iteration estimate of the critical load. The application of this method to the contact problems in combination with the

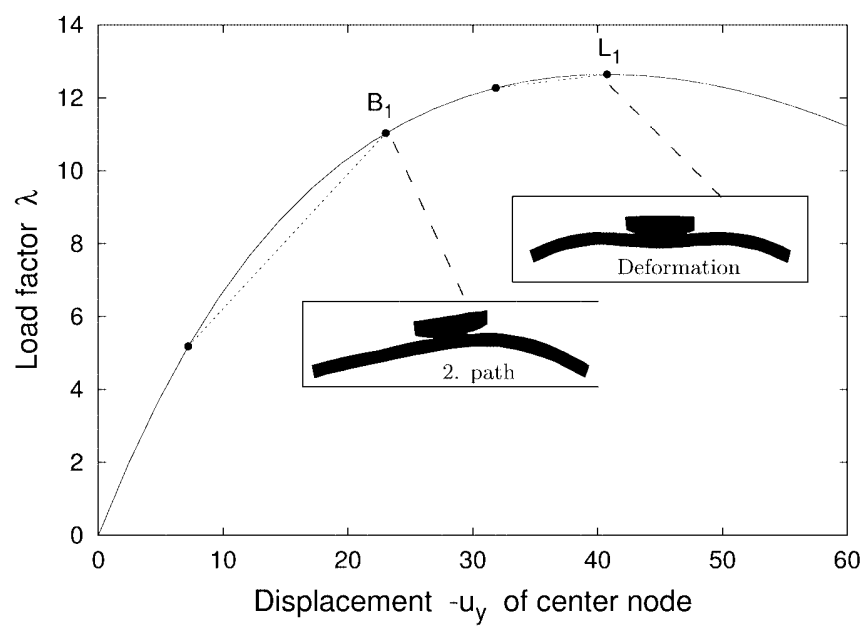

Fig. 6. Equilibrium path for the block pressing on hinged arch

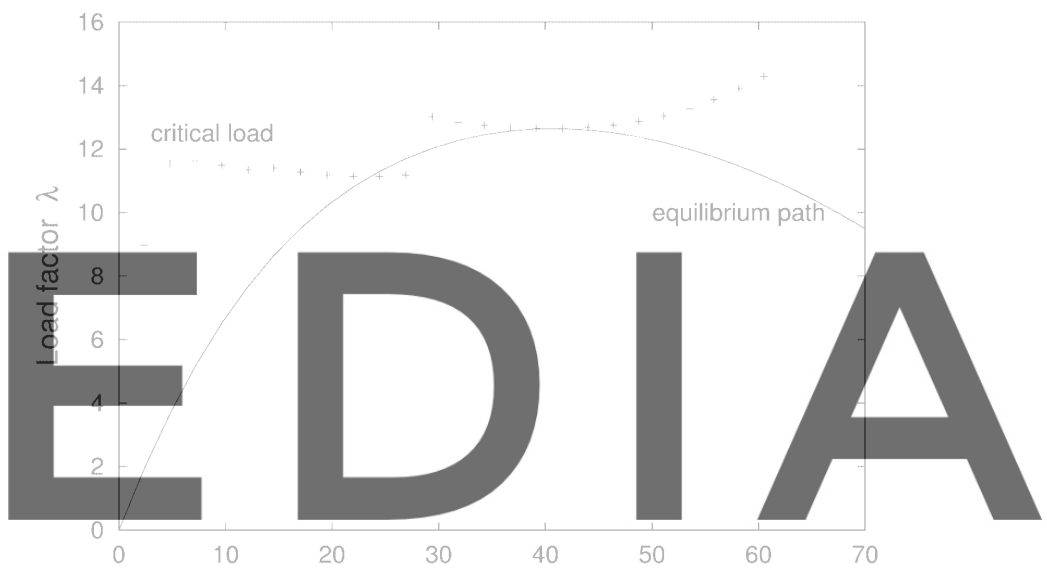

download the visolacementiou of of withor noute the watermark Fig. 7. One step predictions for the block pressing on hinged arch

path computation showed that the predicted critical loads can serve as an indicator for good starting values of the extended system.

\section{Appendix A \\ Partitioning algorithm for the extended system}

Solve for $\Delta \mathbf{v}^{i+1}$

$\left[\mathbf{K}_{T}\left(\mathbf{v}^{i}\right)+\mathbf{K}_{c}\left(\mathbf{v}^{i}\right)\right]_{\Delta} \mathbf{v}_{P}^{i+1}=\mathbf{P}$

$\left[\mathbf{K}_{T}\left(\mathbf{v}^{i}\right)+\mathbf{K}_{c}\left(\mathbf{v}^{i}\right)\right]_{\Delta} \mathbf{v}_{G}^{i+1}=-\mathbf{G}_{S}\left(\mathbf{v}^{i}\right)-\mathbf{G}_{c}\left(\mathbf{v}^{i}\right)$

Compute

$\mathbf{h}_{1}^{i}=\frac{1}{\epsilon}\left[\mathbf{K}_{T}\left(\mathbf{v}^{i}+\epsilon \boldsymbol{\phi}^{i}\right)_{\Delta} \mathbf{v}_{P}^{i+1}+\mathbf{K}_{c}\left(\mathbf{v}^{i}+\epsilon \boldsymbol{\phi}^{i}\right)_{\Delta} \mathbf{v}_{P}^{i+1}-\mathbf{P}\right]$

$\mathbf{h}_{2}^{i}=\frac{1}{\epsilon}\left[\mathbf{K}_{T}\left(\mathbf{v}^{i}+\epsilon \boldsymbol{\phi}^{i}\right)_{\Delta} \mathbf{v}_{G}^{i+1}+\mathbf{K}_{c}\left(\mathbf{v}^{i}+\epsilon \boldsymbol{\phi}^{i}\right)_{\Delta} \mathbf{v}_{G}^{i+1}\right.$

$$
\left.+\mathbf{G}_{S}\left(\mathbf{v}^{i}\right)+\mathbf{G}_{c}\left(\mathbf{v}^{i}\right)\right]
$$

Solve for ${ }_{\Delta} \phi^{i+1}$ 


$$
\begin{aligned}
& {\left[\mathbf{K}_{T}\left(\mathbf{v}^{i}\right)+\mathbf{K}_{c}\left(\mathbf{v}^{i}\right)\right]_{\Delta} \boldsymbol{\phi}_{1}^{i+1}=-\mathbf{h}_{1}^{i}} \\
& {\left[\mathbf{K}_{T}\left(\mathbf{v}^{i}\right)+\mathbf{K}_{c}\left(\mathbf{v}^{i}\right)\right]_{\Delta} \boldsymbol{\phi}_{2}^{i+1}=-\mathbf{h}_{2}^{i}}
\end{aligned}
$$

Compute increments

${ }_{\Delta} \lambda^{i+1}=\frac{-\boldsymbol{\phi}^{i^{\mathrm{T}}}{ }_{\Delta} \boldsymbol{\phi}_{2}^{i+1}+\left\|\boldsymbol{\phi}^{i}\right\|}{\boldsymbol{\phi}^{i^{\mathrm{T}}}{ }_{\Delta} \boldsymbol{\phi}_{1}^{i+1}}$

${ }_{\Delta} \mathbf{v}^{i+1}={ }_{\Delta} \lambda^{i+1}{ }_{\Delta} \mathbf{v}_{P}^{i+1}+{ }_{\Delta} \mathbf{v}_{G}^{i+1}$

178

\section{Update}

$\lambda^{i+1}=\lambda^{i}+{ }_{\Delta} \lambda^{i+1}, \quad \mathbf{v}^{i+1}=\mathbf{v}^{i}+{ }_{\Delta} \mathbf{v}^{i+1}$,

$\phi^{i+1}={ }_{\Delta} \lambda^{i}{ }_{\Delta} \phi_{1}^{i+1}+{ }_{\Delta} \phi_{2}^{i+1}$

\section{References}

Abbott J (1978) An efficient algorithm for the determination of certain bifurcation points. J. Comput. Appl. Math. 4(1): 19-27

Crisfield M (1981) A fast incremental/iterative solution procedure that handles snap through. Comput. Struct. 13: 55-62

Curnier A (1984) A theory of friction. Int. J. Solids Struct. 20: $637-647$

Hallquist J (1979) Nike2d: An implicit finite deformation, finite element code for analyzing the static and dynamic response on two-dimensional solids. Report UCRL-52678, UC-Lawrence Livermore National Laboratory

Jepson A, Spence A (1985) Fo systems and their calculat: 347-369

Laursen T, Simo J (1993) A con formulation for the implicit deformation fricitional conta Meth. Eng. 36: 3451-3485

Oñate E, Matias W (1996) A critical displacenent approach for predicting structural instability. Comput. Meth. Appl. Mech. Eng. 134: 135-161

Oñate E Tschöpe He Wriggers $\mathrm{P}$ (2001) Combination of the structural instability analysis. Eng. Comput. 18(3/4): 642662
Ramm E (1981) Strategies for tracing the nonlinear response near limit points. In: Wunderlich W, Stein E, Bathe K-J (eds) Nonlinear Finite Element Analysis in Structural Mechanics, pp. 63-89. Springer Verlag, Berlin, Heidelberg, New York

Riks E (1972) The application of newtons method to the problem of elastic stability. J. Appl. Mech. 39: 1060-1066

Schweizerhof K, Wriggers P (1986) Consistent linearization for path following methods in nonlinear FE analysis. Comput. Meth. Appl. Mech. Eng. 59: 261-279

Seydel R (1979) Numerical computation of branch points in nonlinear equations. Numerische Mathematik 33: 339-352

Tschöpe H (2001) Direct computation of instability points with inequality constraints using the finite element method. $\mathrm{PhD}$ thesis, Universität Hannover, Institut für Baumechanik und numerische Mechanik

Wagner W (1991) Zur Behandlung von Stabilitätsproblemen der Elastostatik mit der Methode der Finiten Elemente. Forschungs- und Seminarberichte aus dem Bereich der Mechanik der Universität Hannover F 91/1, IBNM, Universität Hannover

Wemper G (1971) Discrete approximation related to nonlinear theories of solids. Int. J. Soilds Struct. 7: 1581-1599

Wriggers P (1995) Finite element algorithms for contact problems. Vol. 2, pp. 1-49. CIMNE, Barcelona

Wriggers P, Miehe C (1992) Recent advances in the simulation of thermomechanical contact processes. In: Owen DRJ, Hinton EO (eds) Proceedings of COMPLAS III, Swansea. Pineridge Press

Wriggers P, Simo J (1990) A general procedure for the direct

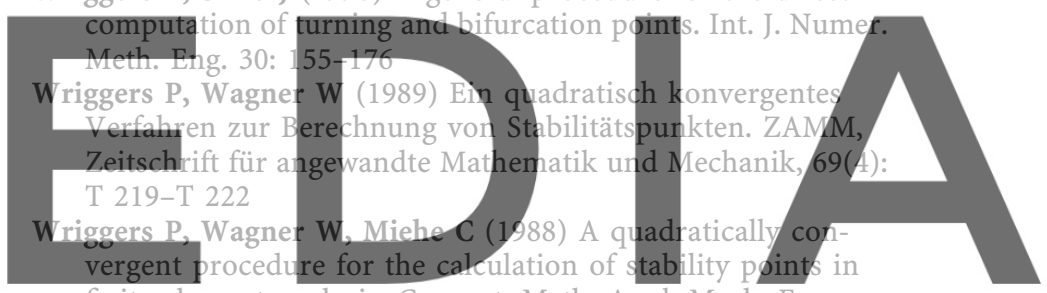
finite element analysis. Comput. Meth. Appl. Mech. Eng. 70: 329-347 